\title{
SirT1 activator represses the transcription of TNF- $\alpha$ in THP-1 cells of a sepsis model via deacetylation of H4K16
}

\author{
GUO-DONG CHEN ${ }^{1}$, WEI-DONG YU ${ }^{2}$ and XIAO-PING CHEN ${ }^{3}$ \\ ${ }^{1}$ Department of Gastroenterology; ${ }^{2}$ Central Laboratory, Peking University People's Hospital, Beijing 100044; \\ ${ }^{3}$ State Key Laboratory for Infectious Disease Prevention and Control, \\ Collaborative Innovation Center for Diagnosis and Treatment of Infectious Diseases, Department of Nosocomial Infection, \\ National Institute for Communicable Disease Control and Prevention, \\ Chinese Center for Disease Control and Prevention, Beijing 102206, P.R. China
}

Received September 19, 2015; Accepted October 12, 2016

DOI: $10.3892 / \mathrm{mmr} .2016 .5942$

\begin{abstract}
Sepsis is a systemic inflammatory response resulting from the excessive production of pro-inflammatory cytokines, including tumor necrosis factor (TNF)- $\alpha$. Sirtuin 1 (SirT1) actively deacetylates histone proteins, and facilitates chromatin compaction and gene silencing. In the present study, a cell model of sepsis, comprising lipopolysaccharide (LPS)-tolerant THP-1 cells, was used to investigate whether the SirT1 activator, resveratrol, repressed the transcription of TNF- $\alpha$. Chromatin immunoprecipitation and real-time PCR were used to determine the transcription of the TNF- $\alpha$ promoter. The result revealed that the binding of SirT1 to the TNF- $\alpha$ promoter was decreased by LPS stimulation in normal cells. However, in LPS-tolerant cells, nuclear protein levels of SirT1 remained elevated, and LPS stimulation had no significant effect on the binding of SirT1 to the TNF- $\alpha$ promoter. However, the activity of SirT1 was increased and binding of ace-H4K16 to the TNF- $\alpha$ promoter was decreased with resveratrol treatment in the tolerant cells. It was concluded that resveratrol stimulated sirtuin activity in LPS-tolerant THP-1 cells, and repressed TNF- $\alpha$ transcription through the deacetylation of H4K16, without affecting the methylation of H3K9. Resveratrol offers potential as an infective candidate to alleviate inflammation in patients with sepsis.
\end{abstract}

Correspondence to: Dr Xiao-Ping Chen, State Key Laboratory for Infectious Disease Prevention and Control, Collaborative Innovation Center for Diagnosis and Treatment of Infectious Diseases, Department of Nosocomial Infection, National Institute for Communicable Disease Control and Prevention, Chinese Center for Disease Control and Prevention, 155 Changbai Road, Changping, Beijing 102206, P.R. China

E-mail: chenxiaoping@icdc.cn

Key words: sepsis, sirtuin 1, resveratrol, tumor necrosis factor- $\alpha$, H4K16 deacetylation

\section{Introduction}

Sepsis is a systemic inflammatory response mediated by various innate immune cells, including neutrophiles, monocytes and macrophages, upon severe infection (1). Normally, the moderate production of pro-inflammatory cytokines, including tumor necrosis factor (TNF)- $\alpha$, interleukin (IL)-1, IL-6 and IL-8 assist in confining infection and tissue damage; with the eradication of infectious agents, the inflammatory response can recover to homeostasis. However, excessive and prolonged production of inflammatory cytokines can lead to an overwhelming inflammatory response, which is referred to as sepsis (2). The mortality rates of severe sepsis can reach as high as $70 \%$ and the number of cases of sepsis continues to increase due to the continued increase in the number of immunocompromised patients $(3,4)$. However, the molecular mechanism of sepsis remains to be fully elucidated. Studies have revealed that several mechanisms may contribute to the occurrence of sepsis, including the continued activation of neutrophils and macrophages/monocytes, upregulation of lymphocyte costimulatory molecules $(5,6)$, rapid lymphocyte apoptosis and delayed neutrophil apoptosis, and excessive necrosis of cells and tissues $(7,8)$. Of these, the overwhelming production of TNF- $\alpha$ is considered to be important in the occurrence and development of sepsis (9).

Sirtuin 1 (sirT1), an $\mathrm{NAD}^{+}$-dependent deacetylase, has been well-established as a major component in the regulation of cellular stress responses, including apoptosis, autophagic DNA damage repair and metabolic disorders through histone and non-histone deacetylation. It is not surprising that SirT1 has a number of roles in multiple tissues through its effects on diverse physiological processes $(10,11)$. Previous studies have further revealed the role of SirT1 in sepsis. For example, nuclear SirT1 has been found to guide RelB to promote mitochondrial biogenesis, which alters bioenergetics during sepsis adaptation (12). Resveratrol, an activator of SirT1, has been revealed to protect against sepsis-induced liver injury through promoting the SirT1-mediated nucleocytoplasmic translocation of high mobility group box 1 (HMGB1) (13). Other previous studies have examined the association between SirT1 and inflammatory cytokines in sepsis. For example, 
acute hyperglycemia in sepsis is considered to repress the transcription and translation of SirT1, and then promote the transcription and translation of TNF- $\alpha$ and IL-1 $\beta$ (14). Studies have shown that SirT1 inhibits acute lung inflammation during sepsis by repressing the inflammasome activation pathway, including the activation of nuclear factor $(\mathrm{NF})-\kappa \mathrm{B}$, signal transducer and activator of transcription 3 and extracellular signal-regulated kinase (ERK)1/2 (15). In particular, the NF- $\kappa \mathrm{B}$ transcription factor, RelA/p65, is considered to be the primary target of SirT1 in regulating the transcription of TNF- $\alpha$ through deacetylating RelA/p65 in innate cells $(16,17)$.

It is well-known that SirT1 is also involved in chromatin compaction and gene silencing through deacetylating H4K16, H3K9 and H1K26 $(18,19)$. Studies have also shown that SirT1 deacetylates H4K16 and promotes termination of the NF- $\kappa \mathrm{B}$-dependent transcription of TNF- $\alpha$ during initial lipopolysaccharide (LPS) stimulation in normal THP-1 cells (20). However, the exact role of SirT1 in epigenetic modifications of inflammatory gene promoters in sepsis remains to be fully elucidated. In the present study, epigenetic modifications by SirT1 and resveratrol were assayed in a cell model of sepsis, namely LPS-mediated tolerance in THP-1 promonocytes (21), to reveal whether SirT1 activation can repress the transcription of TNF- $\alpha$ in the sepsis model, and offer potential as a promising candidate for sepsis therapy.

\section{Materials and methods}

Cell culture model of sepsis and resveratrol treatment. THP-1 cells, obtained from the American Type Culture Collection (Manassas, VA, USA) were maintained in Gibco RPMI-1640 medium (Thermo Fisher Scientific, Inc., Waltham, MA, USA) supplemented with $10 \mathrm{U} / \mathrm{ml}$ penicillin $\mathrm{G}, 10 \mu \mathrm{g} / \mathrm{ml}$ streptomycin, 2 mM L-glutamine and 10\% FBS (HyClone; GE Healthcare Life Sciences, Logan, UT, USA) at $37^{\circ} \mathrm{C}$ and $5 \% \mathrm{CO}_{2}$ in a humidified incubator. The sepsis phenotype (LPS-tolerant) was mimicked by stimulation of the THP-1 cells with LPS (0111:B4; $1 \mu \mathrm{g} / \mathrm{ml})$ overnight (21). The normal and LPS-tolerant group THP-1 cells ( $1 \times 10^{6}$ cells/sample) were washed once with RPMI-1640, re-suspended in FBS supplemented RPMI-1640 medium at $1 \times 10^{6}$ cells $/ \mathrm{ml}$, and stimulated by $1 \mu \mathrm{g} / \mathrm{ml}$ LPS (Sigma-Aldrich; Merck Millipore, Darmstadt, Germany) for $3 \mathrm{~h}$ at $37^{\circ} \mathrm{C}$ and $5 \% \mathrm{CO}_{2}$. The normal+resveratrol group and tolerant+resveratrol group were treated with resveratrol (10 $\mu \mathrm{mol} / 1$; Sigma-Aldrich; Merck Millipore) (22) for $30 \mathrm{~min}$ at $37^{\circ} \mathrm{C}$ and $5 \% \mathrm{CO}_{2}$ prior to the LPS stimulation described above.

Chromatin immunoprecipitation (ChIP) assay. To assess the binding of SirT1, H3K9me2 and H4K16ace around the $\mathrm{NF}-\kappa \mathrm{B}$ binding site of the TNF- $\alpha$ proximal promoter in the SirT1-treated tolerant THP-1 cells, ChIP assays (Upstate Biotechnology, Inc., Lake Placid, NY, USA) were performed with the following modifications. Proteins from $5 \times 10^{6}$ cells in each sample were cross-linked to DNA using $1 \%$ formaldehyde for $10 \mathrm{~min}$ at room temperature. Each sample with sheared chromatin was divided into two sample groups, providing an 'input' sample, which was not incubated with any antibodies. The other sample was incubated with antibodies specific for
SirT1 (cat. no. sc-135792; 1:500; Santa Cruz Biotechnology, Inc., Santa Cruz, CA, USA), H3K9me2 (cat. no. 6814-25; 1:600; BioVision, Inc., Milpitas, CA, USA), H4K16ace (cat. no. sc-8662; 1:500; Santa Cruz Biotechnology, Inc.), and IgG (1:800; cat. no. sc-2027; Santa Cruz Biotechnology, Inc.) for the negative control at $4^{\circ} \mathrm{C}$ overnight.

Reverse transcription-quantitative polymerase chain reaction (RT-qPCR) analysis. The ChIP DNA for each treatment group were analyzed quantitatively through the amplification of a sequence in the human TNF- $\alpha$ proximal promoter region containing the $\kappa \mathrm{B} 3$ site at $-98 \mathrm{bp}$, relative to the transcription start site (23). The primers were as follows: Forward, 5'TAC CGCTTCCTCCAGATGAG-3' and reverse, 5'-TGCTGGCTG GGTGTGCCAA-3'. The probe was: 5'-6-FAM TTGGTGGAG AAACC-TAMRA-3' (Sangon Biotech Co., Ltd., Shanghai, China). The PCR reaction (total $20 \mu \mathrm{l}$ ) contained $2 \mu \mathrm{l}$ DNA, $10 \mu 1$ 2x TaqMan Universal Master Mix, $300 \mathrm{nM}$ each primer and $100 \mathrm{nM}$ dNTPs. The PCR procedure was as follows: $2 \mathrm{~min}$ at $50^{\circ} \mathrm{C}, 10 \mathrm{~min}$ at $95^{\circ} \mathrm{C}$, followed by 40 cycles with $15 \mathrm{sec}$ at $95^{\circ} \mathrm{C}$ and $15 \mathrm{sec}$ at $60^{\circ} \mathrm{C}$, using an ABI 7500 fast detection system (Thermo Fisher Scientific, Inc.). Data were normalized to the input DNA and are presented as the fold change, relative to DNA from the untreated cells.

To measure the mRNA expression of TNF- $\alpha$, total RNA was isolated from cells using a Qiagen RNA mini kit (Qiagen, Inc., Valencia, CA, USA) according to the manufacturer's protocol. The RNA ( $1 \mu \mathrm{g})$ was reverse transcribed into cDNA in a $20 \mu \mathrm{l}$ volume containing $5 \mathrm{mM} \mathrm{MgCl}, 1 \mathrm{mM} \mathrm{dNTP}$, $2.5 \mu \mathrm{M}$ Oligo d (T), $2.5 \mathrm{U} / \mu 1 \mathrm{MuLV}$ Reverse Transcriptase (Thermo Fisher Scientific, Inc.). The qPCR was performed using $3 \mu \mathrm{l}$ cDNA TNF- $\alpha$ and GAPDH predesigned TaqMan primer/probe sets (Thermo Fisher Scientific, Inc.) under the conditions described above. Data were normalized to GAPDH mRNA, which was analyzed using GAPDH pre-designed TaqMan primer/probe kits (Thermo Fisher Scientific, Inc.) and are presented as the fold change, relative to mRNA from the untreated cells. Sample data were normalized to GAPDH mRNA values and are presented as the fold changes, relative to mRNA from the untreated cells (23).

PCR analysis. A standard PCR reaction (total $25 \mu \mathrm{l}$ ) was composed of $2 \mu \mathrm{l}$ ChIPed DNA or $2 \mu \mathrm{l}$ Input DNA, $1 \mu \mathrm{M}$ of each primer (as above), $2 \mathrm{mM} \mathrm{MgCl} \mathrm{m}_{2}, 0.2 \mathrm{M}$ dNTPs and $0.03 \mathrm{U} / \mu 1$ AmpliTaq Gold DNA polymerase (Thermo Fisher Scientific, Inc.) was performed to confirm the results of the RT-qPCR analysis. The PCR conditions were as follows: 1 cycle at $94^{\circ} \mathrm{C}$ for $5 \mathrm{~min}, 30$ cycles at $94^{\circ} \mathrm{C}, 58^{\circ} \mathrm{C}$ and $72^{\circ} \mathrm{C}$ for $30 \mathrm{sec}$ each, and a final cycle at $72^{\circ} \mathrm{C}$ for $5 \mathrm{~min}$. Equal volumes of PCR product was visualized using $1.5 \%$ agarose gels and images were captured using Quantity One Imager version 4.6.2 (Bio-Rad Laboratories, Inc., Hercules, CA, USA).

SirT1 histone deacetylase (HDAC) activity assay. SirT1 activity was assayed according to a previous study (24). Briefly, the THP-1 cells were homogenized using a Diagenode Bioruptor sonicator (Tosho Denki Co., Ltd., Tokyo, Japan). Total protein was extracted from $1.5 \times 10^{6}$ cells/sample (21). Briefly, cells were collected and washed twice with PBS, resuspended in $100 \mu \mathrm{l}$ RIPA buffer (cell lysis buffer containing $0.45 \% \mathrm{NaCl}$, 
$0.5 \%$ deoxycholate, $0.5 \%$ Triton X-100, $0.05 \%$ sodium dodecyl sulfate, $0.005 \mathrm{M}$ Tris) and incubated on ice for $20 \mathrm{~min}$. Next, the cells were vortexed for $10 \mathrm{sec}$ and centrifuged at 12,000 $\mathrm{x} g$ for 5 min, supernatant was retained. Protein quantity was assayed with Bradford method protein assay kit (Amresco, LLC, Solon, OH, USA). Each sample (containing $30 \mu \mathrm{g} / 10 \mu \mathrm{l}$ total protein) was used for the measurement of SirT1 activity. The activity of HDAC was determined using an SirT1 Fluorimetric Drug Discovery kit (Enzo Life Sciences, Inc., Farmingdale, NY, USA) according to the manufacturer's protocol. The THP-1 cell protein extracts were incubated in assay buffer with $\beta$-nicotinamide adenine dinucleotide $\left(\mathrm{NAD}^{+}\right)$substrate at $37^{\circ} \mathrm{C}$ for $45 \mathrm{~min}$. The fluorescence density was determined using a multimode detector (DTx880; Beckman Coulter, Brea, CA). The SirT1 activity was determined, relative to that in the untreated control cells.

Western blot analysis. Total nuclear protein was assayed using western blot analysis. The methods for protein extraction are the same as described previously (21). Whole-cell protein $(20 \mu \mathrm{g})$ or nuclear protein $(30 \mu \mathrm{g})$ was separated by SDS-PAGE and transferred to PVDF membranes. SirT1 antibodies (cat no. sc-135791; 1:800; Santa Cruz Biotechnology, Inc.) were used to visualize and quantify protein levels following incubation at $37^{\circ} \mathrm{C}$ for $30 \mathrm{~min}$ using Image Quant software 4.6.2 (GE Healthcare Life Sciences). IgG (cat. no. sc-69917; 1:1,000) was used as negative control.

\section{Results}

Resveratrol treatment suppresses the $m R N A$ transcription of TNF- $\alpha$ in LPS-tolerant THP-1 cells. To assess whether resveratrol treatment repressed the mRNA transcription of TNF- $\alpha$ in a cell model of sepsis, RT-qPCR analysis was used to assess the mRNA levels of TNF- $\alpha$. As indicated in Fig. 1, the mRNA levels of TNF- $\alpha$ rapidly and markedly increased in the normal cells stimulated with LPS, and then reduced over the $4 \mathrm{~h}$ period. By contrast, in the tolerant cells, the peak increase in the mRNA transcription of TNF- $\alpha$ was relatively lower following LPS re-stimulation, compared with the normal cells, which indicated that the sepsis model using THP-1 cells had been successfully established. Following resveratrol treatment for 30 mins, the mRNA levels of TNF- $\alpha$ decreased significantly in the tolerant+resveratrol group, compared with the tolerant group, particularly at the $1 \mathrm{~h}$ time point. In the normal cells, resveratrol treatment also suppressed the mRNA transcription of TNF- $\alpha$, as indicated in the normal+resveratrol group, compared with the normal group.

Protein levels of SirT1 are not altered significantly with resveratrol treatment in tolerant cells. To analyze whether resveratrol treatment affected the protein transcription and translation of SirT1, western blot analysis was used to measure the protein levels of SirT1 in the THP-1 cells of each group $0,0.5,1$ and $3 \mathrm{~h}$ following stimulation by LPS. As shown in Fig. 2, nuclear SirT1 protein decreased and then recovered partially in the normal and normal+resveratrol groups following LPS stimulation. However, the nuclear protein levels of SirT1 remained substantially higher over the $4 \mathrm{~h}$ assessment

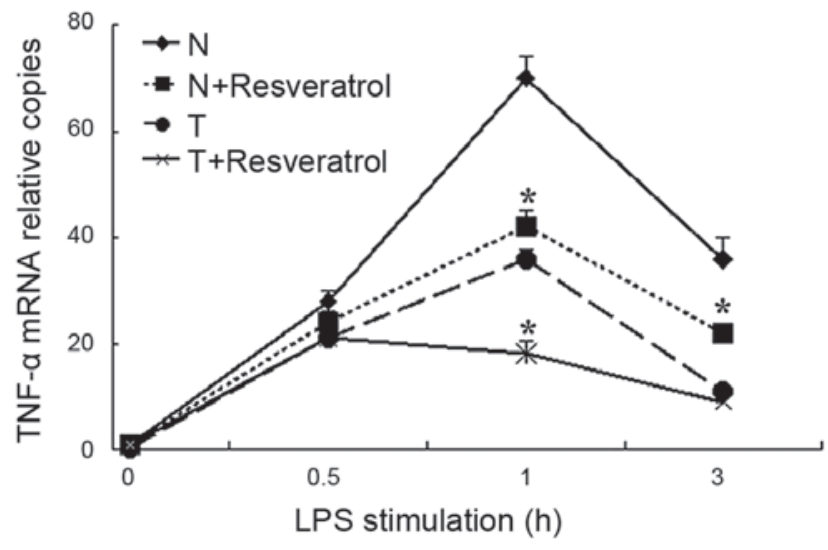

Figure 1. Resveratrol treatment alleviates the mRNA transcription of TNF- $\alpha$ in THP-1 cells induced by LPS stimulation. The mRNA level of TNF- $\alpha$ in the T+resveratrol group was significantly lower, compared with that in the T group, particularly at $1 \mathrm{~h}$. The mRNA level of TNF- $\alpha$ in the $\mathrm{N}+$ resveratrol group was significantly lower, compared with levels in the $\mathrm{N}$ group at 1 and $3 \mathrm{~h}$. Data are presented as the mean \pm standard error of the mean from three independent experiments and are presented as the increase in copy number relative to that at $0 \mathrm{~h}$ (set as an arbitrary unit of one) ${ }^{*} \mathrm{P}<0.05$ vs. $\mathrm{N}$ group. $\mathrm{N}$, normal; T, tolerant; TNF- $\alpha$, tumor necrosis factor- $\alpha$; LPS, lipopolysaccharide.

period in the tolerant group and tolerant+resveratrol group. In addition, the nuclear protein levels of SirT1 in the tolerant group and tolerant+resveratrol groups were significantly higher, compared with those in the normal and normal+resveratrol groups. Of note, the whole cell protein levels of SirT1 in the normal+resveratrol group, tolerant group and tolerant+resveratrol group were higher, compared with that in the normal group. However, it was clear that resveratrol treatment had no effect on the protein levels of SirT1 in the tolerant cells.

Resveratrol treatment promotes SirT1 activity and the binding of SirT1 to the TNF- $\alpha$ promoter in tolerant cells. To investigate the effect of resveratrol on SirT1 activity, THP-1 cells in each group were collected at 0 and $2 \mathrm{~h}$-post LPS stimulation, and activities of SirT1 HDAC were analyzed (Fig. 3). Although the activities of SirT1 in the normal cells (N group) were reduced $2 \mathrm{~h}$-post LPS stimulation, the decreases were not statistically significant. No significant alterations were found in the activities of SirT 1 in the normal cells or tolerant cells at $2 \mathrm{~h}$-post LPS stimulation. However, the activity of SirT1 in the tolerant cells was higher, compared with that in the normal cells. Following resveratrol treatment, the activities of SirT1 were increased significantly in the normal cells and tolerant cells. To further assess the binding of SirT1 to the TNF- $\alpha$ promoter $\kappa$ B3 site at the core promoter, the functionally important NF- $\mathrm{\kappa B}$ site for the activation of TNF- $\alpha$ transcription (23), ChIP assays were used. It was shown that, in the normal group, binding of SirT1 to the TNF- $\alpha$ promoter was decreased $2 \mathrm{~h}$ post-LPS stimulation (Fig. 4), whereas binding of SirT1 was increased significantly in the normal+resveratrol group. In the tolerant group, the binding of SirT1 remained consistently higher, compared with that in the normal cells under LPS stimulation, and resveratrol promoted the binding of SirT1 to the TNF- $\alpha$ promoter, as indicated in the tolerant+resveratrol group. 


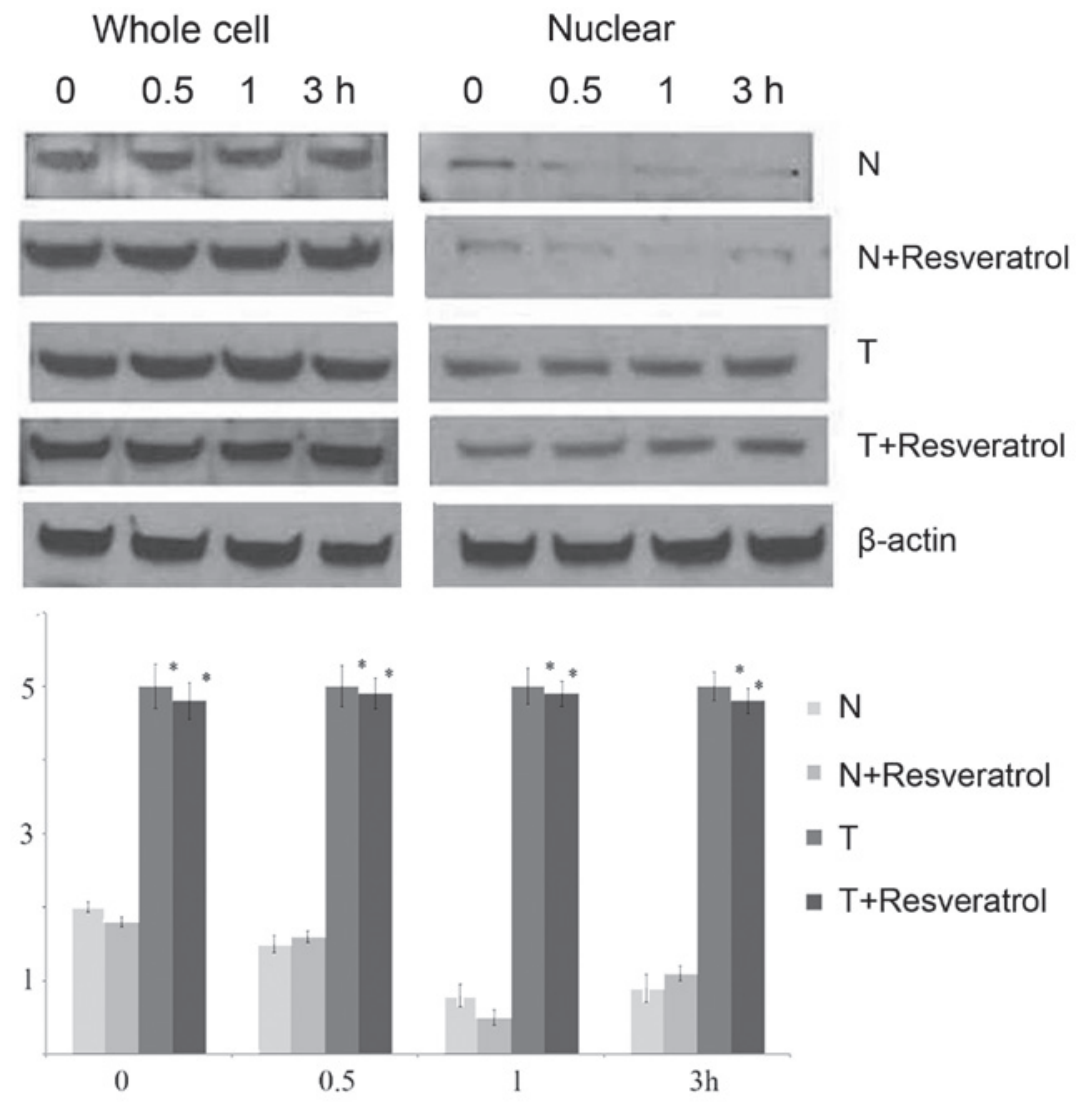

Figure 2. Resveratrol treatment has no significant effect on protein levels of SirT1 in tolerant cells. In the T group of THP-1 cells, resveratrol did not affect protein levels of SirT1 in the cytosol (left) or nucleus (right). In the N group of cells, whole cell protein levels of SirT1 increased following resveratrol treatment. Nuclear protein levels of SirT1 decreased initially and then partially recovered in the $\mathrm{N}$ and N+resveratrol groups. In the $\mathrm{T}$ and $\mathrm{T}+\mathrm{resveratrol}$ groups, nuclear protein levels of SirT1 were sustained at a high level. "P<0.05. N, normal; T, tolerant; SirT1, sirtuin 1.

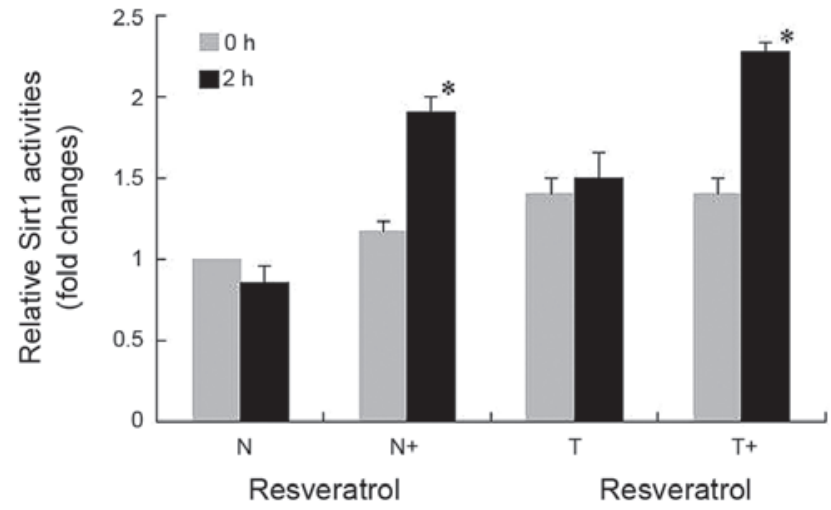

Figure 3. Resveratrol treatment promotes SirT1 activity. Analysis of the activity of SirT1 demonstrated that resveratrol treatment increased the activity of SirT1 substantially in the $\mathrm{N}$ and $\mathrm{T}$ cells. ${ }^{*} \mathrm{P}<0.05$ vs. $\mathrm{N}$ group. $\mathrm{N}$ normal; T, tolerant; SirT1, sirtuin 1.

Binding of H4K16ace to the TNF- $\alpha$ promoter decreases with resveratrol treatment in tolerant cells. The epigenetic regulation in the TNF- $\alpha$ promoter was assessed using ChIP assays to analyze the binding of $\mathrm{H} 4 \mathrm{~K} 16$ ace and $\mathrm{H} 3 \mathrm{~K} 9 \mathrm{me} 2$ to the $\kappa \mathrm{B} 3$ site (Fig. 5). In the normal group, H4K16ace was increased and $\mathrm{H} 3 \mathrm{~K} 9 \mathrm{me} 2$ was decreased at $2 \mathrm{~h}$ post-LPS stimulation. In the normal+resveratrol group, H4K16ace binding decreased with an accompanied increase in $\mathrm{H} 3 \mathrm{~K} 9 \mathrm{me} 2$ binding at $2 \mathrm{~h}$ post-LPS stimulation. In the tolerant cells, prominent H4K16ace and
H3K9me2 binding to the TNF- $\alpha$ promoter was sustained constantly regardless of the time course of LPS stimulation, whereas H4K16ace binding decreased significantly with resveratrol treatment (tolerant+resveratrol group) without effects on H3K9me2 binding.

\section{Discussion}

In the present study, the SirT1 activator, resveratrol, successfully repressed the transcription of TNF- $\alpha$ through deacetylating $\mathrm{H} 4 \mathrm{~K} 16$ at the TNF- $\alpha$ promoter, which indicated that resveratrol may be a promising therapeutic candidate for sepsis. Human SirT1 consists of 747 amino acids, divided into four major regions: N-terminal domain (residues 1-182), allosteric site (residues 183-243), catalytic core (residues 244-498) and C-terminal domain (residues 499-747) (25). SirT1 catalyzes the protein deacetylation reaction in its catalytic core, which consists of two subdomains for $\mathrm{NAD}^{+}$and substrate binding (26). Adjacent to the N-terminal in the catalytic core is the compacted allosteric domain, to which SirT1 activators, including resveratrol, bind and positively regulate sirtuin activity (27). Resveratrol has been shown to activate SirT1 and suppress the overexpression of pro-inflammatory molecules in a dose-dependent manner in a mouse model of sepsis induced by LPS (28). The results of the present further supported that resveratrol decreased the transcription of TNF- $\alpha$ under LPS stimulation in tolerant cells and in normal cells. 
Input ChIP
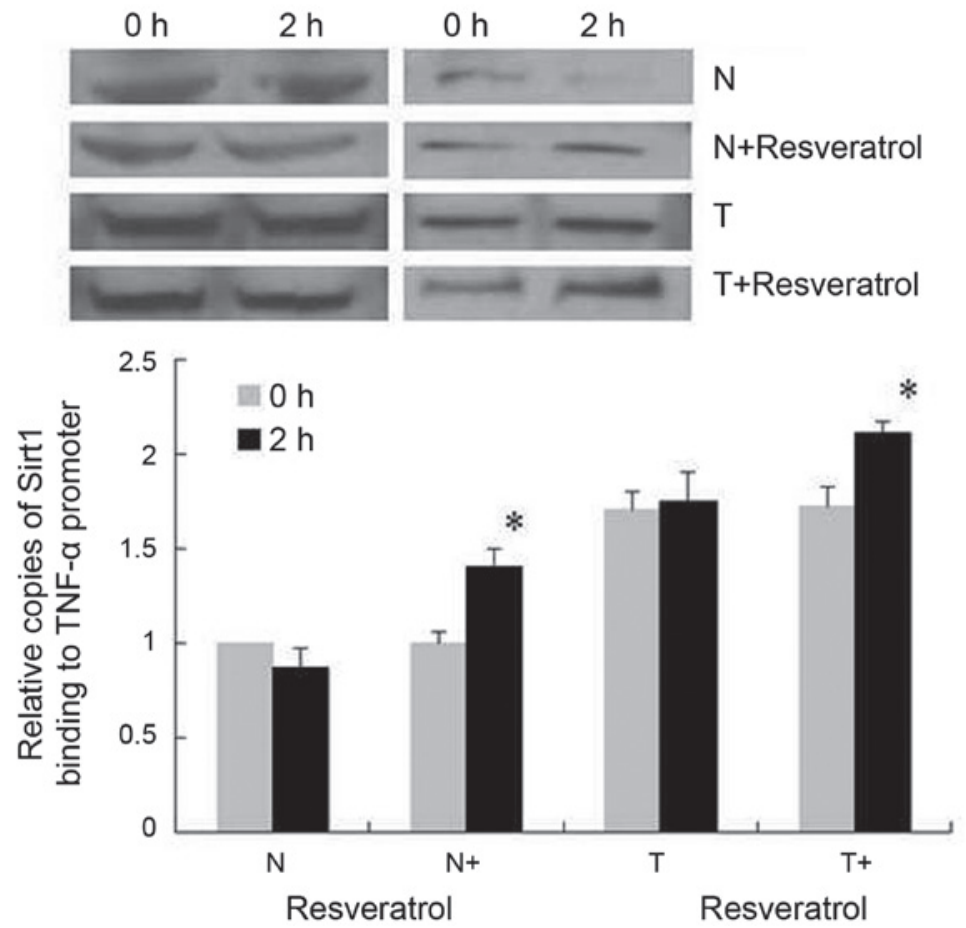

Figure 4. Resveratrol treatment increases the binding of SirT1 to the TNF- $\alpha$ promoter in N and T cells. Results of the PCR analysis (above) and RT-qPCR analysis (below) are shown. ChIP analyses indicated that the binding of SirT1 to the TNF- $\alpha$ promoter increased at $2 \mathrm{~h}$ in the N and T cells. Data for RT-qPCR are presented as the mean \pm standard error of the mean of three independent experiments and are presented as the increase in binding relative to that of $\mathrm{N}$ cells at $0 \mathrm{~h}$ (arbitrary unit of one). ${ }^{*} \mathrm{P}<0.052 \mathrm{~h}$ group vs $0 \mathrm{~h}$ group. The standard PCR results of the ChIP samples with the same primer sets as in the RT-qPCR are representative of three independent experiments. PCR, polymerase chain reaction; N, normal; T, tolerant; SirT1, sirtuin 1; TNF- $\alpha$, tumor necrosis factor- $\alpha$.
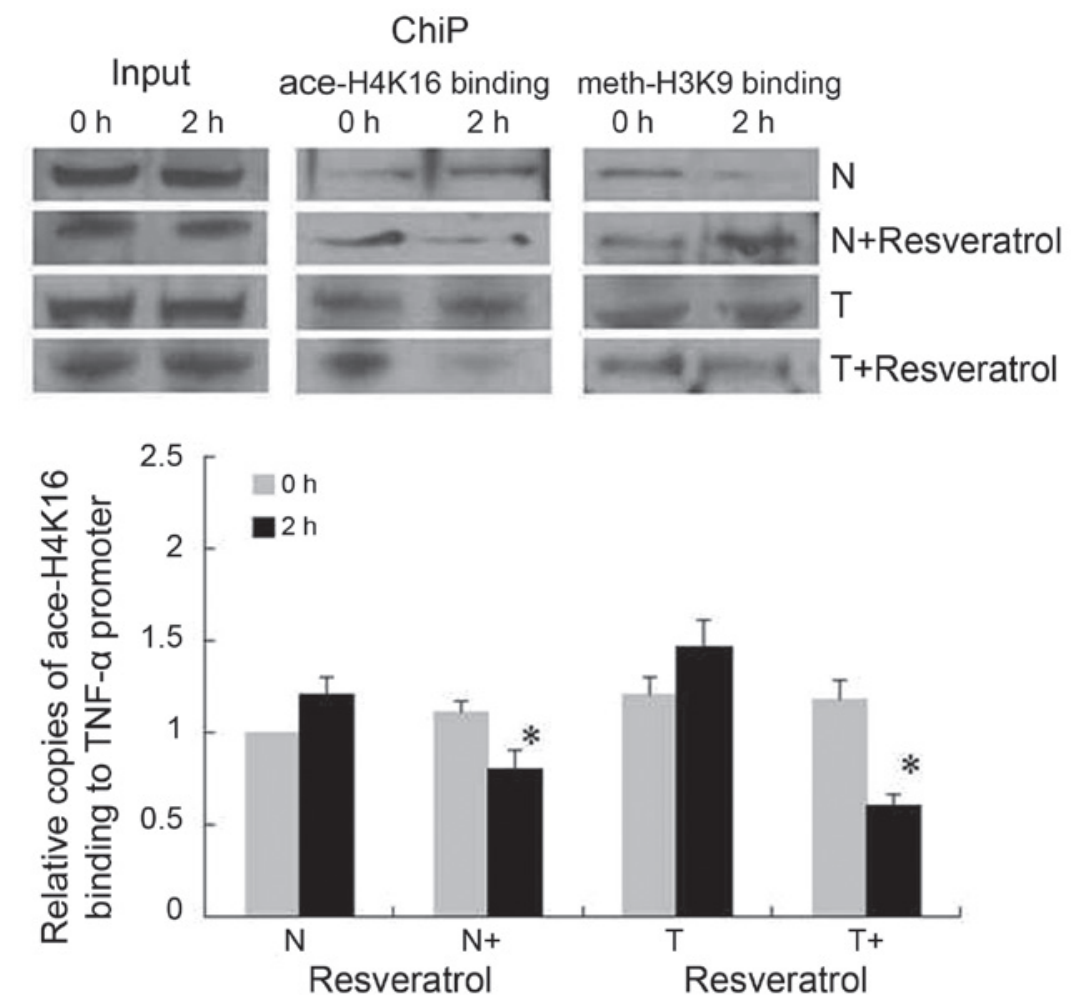

Figure 5. Resveratrol treatment decreases the binding of ace-H4K16 to the TNF- $\alpha$ promoter in N and T cells. Results of PCR (above) and RT-qPCR (below) analyses are shown. ChIP analyses indicated that the binding of ace-H4K16 decreased in the N+resveratrol and T+resveratrol groups at $2 \mathrm{~h}$. However, in the $\mathrm{T}$ cells, the binding of meth-H3K9 to the TNF- $\alpha$ promoter remained unaltered at $2 \mathrm{~h}$. Data for the RT-qPCR are presented as the mean \pm standard error of the mean of three independent experiments and are presented as the increase in binding relative to that of $\mathrm{N}$ cells at $0 \mathrm{~h}$ (arbitrary unit of one). ${ }^{*} \mathrm{P}<0.052 \mathrm{~h}$ group vs $0 \mathrm{~h}$ group. Standard PCR results of ChIP samples with the same primer sets are representative of three independent experiments. PCR, polymerase chain reaction; N, normal; T, tolerant; SirT1, sirtuin 1; TNF- $\alpha$, tumor necrosis factor- $\alpha$; ace, acetylated; meth, methylated. 
A previous study indicated that the protein levels of SirT1 decrease transiently, followed by a substantial increase between 8 and $24 \mathrm{~h}$ of LPS stimulation, in THP-1 cells, which is attributed to increased SirT1 protein synthesis in tolerant cells (20). The present study also found higher protein levels of SirT1 in tolerant cells, compared with normal cells. However, rather than analyzing alterations in whole cell SirT1 protein, the present study assessed the levels of nuclear SirT1 protein in each group. In normal THP-1 cells, the level of nuclear SirT1 protein decreased initially, and then recovered partially under LPS stimulation, which was similar to the observations in whole cell SirT1 protein reported previously (20). It is possible that nuclear SirT1 repressed the transcription of inflammatory genes in basal conditions by repressing TNF- $\alpha$ promoter transcription. Under LPS stimulation, nuclear SirT1 decreased, possibly through transferring to the cytosol, indicated by the fact that no significant alterations were found in total SirT1 protein in the present study. In accordance with this, the transcription of TNF- $\alpha$ was increased.

In addition to activating SirT1, resveratrol has been revealed to increase the mRNA and protein levels of SirT1 in Wistar rats, although the mechanism remains to be elucidated (29). In the present study, elevated protein levels of SirT1 were observed in normal cells treated with resveratrol, compared with untreated cells. However, resveratrol appeared to have no effect on the protein level of SirT1 in tolerant cells. These results suggested that, in the tolerant cells, the transcription and translation of SirT1 may have peaked and responded minimally to resveratrol treatment. Accordingly, the nuclear protein level of SirT1 in the LPS-tolerant cells was sustained at high levels, and binding of SirT1 to the TNF- $\alpha$ promoter was more marked, compared with that in normal cells in the present study. This indicated that SirT1 constantly repressed the transcription of TNF- $\alpha$ in tolerant cells, which showed a high mRNA level of TNF- $\alpha$, but were hyporesponsive to LPS re-stimulation (30).

Studies have revealed that SirT1 limits inflammation through several non-histone proteins. It has been reported that SirT1 deacetylates H4K16 and promotes silencing of the transcription of TNF- $\alpha$ on initial LPS stimulation in normal THP-1 cells (20). The present study demonstrated that SirT1 was involved in epigenetic modifications in the TNF- $\alpha$ promoter of cells in sepsis. The binding and activity of SirT1 at the TNF- $\alpha$ promoter were increased in tolerant cells, compared with normal cells. Resveratrol treatment further promoted the activity and binding of SirT1 to the TNF- $\alpha$ promoter in the tolerant cells. As expected, H3K9 methylation decreased with LPS stimulation in normal cells due to chromatin relaxation and gene transcription (31). Resveratrol appeared to repress the transcription of TNF- $\alpha$ through promoting $\mathrm{H} 3 \mathrm{~K} 9$ methylation in normal cells. However, resveratrol had no effect on H3K9 methylation in tolerant cells, which already had higher levels of $\mathrm{H} 3 \mathrm{~K} 9$ methylation at the TNF- $\alpha$ promoter, compared with normal cells. Of note, the contradictory finding of the coexistence of a high level of H4K16 acetylation and H3K9 methylation in the TNF- $\alpha$ promoter in tolerant cells demonstrated that pro- and anti-inflammatory activities were active at the same time. These results indicated that resveratrol further promoted the activity of SirT1 in LPS-tolerant THP-1 cells and repressed the transcription
TNF- $\alpha$ through the deacetylation of H4K16 without affecting the methylation of H3K9. Taken together, the results of the present study indicated that resveratrol, as an activator of SirT1, offers potential as an infective subsidiary treatment to alleviate inflammation in patients with sepsis.

\section{Acknowledgements}

This study was supported by the National Natural Science Foundation of China (grant no. 81201250) and the National Key Technology Support Program (grant no. 2012BAI11B05).

\section{References}

1. Stearns-Kurosawa DJ, Osuchowski MF, Valentine C, Kurosawa S and Remick DG: The pathogenesis of sepsis. Annu Rev Pathol 6: 19-48, 2011.

2. Cai B, Deitch EA and Ulloa L: Novel insights for systemic inflammation in sepsis and hemorrhage. Mediators Inflamm 2010: 642462, 2010.

3. Russell JA: Management of sepsis. N Engl J Med 355: 1699-1713, 2006.

4. Matsuda A, Jacob A, Wu R, Aziz M, Yang WL, Matsutani T, Suzuki H, Furukawa K, Uchida E and Wang P: Novel therapeutic targets for sepsis: Regulation of exaggerated inflammatory responses. J Nippon Med Sch 79: 4-18, 2012.

5. Nolan A, Weiden M, Kelly A, Hoshino Y, Hoshino S, Mehta N and Gold JA: CD40 and CD80/86 act synergistically to regulate inflammation and mortality in polymicrobial sepsis. Am J Respir Crit Care Med 177: 301-308, 2008.

6. Flohé SB, Agrawal H, Schmitz D, Gertz M, Flohé S and Schade FU: Dendritic cells during polymicrobial sepsis rapidly mature but fail to initiate a protective Th1-type immune response. J Leukoc Biol 79: 473-481, 2006.

7. Roger PM, Hyvernat H, Ticchioni M, Kumar G, Dellamonica J and Bernardin G: The early phase of human sepsis is characterized by a combination of apoptosis and proliferation of $\mathrm{T}$ cells. J Crit Care 27: 384-393, 2012.

8. Paunel-Görgülü A, Kirichevska T, Lögters T, Windolf J and Flohé S: Molecular mechanisms underlying delayed apoptosis in neutrophils from multiple trauma patients with and without sepsis. Mol Med 18: 325-335, 2012.

9. Russell JA, Boyd J, Nakada T, Thair S and Walley KR: Molecular mechanisms of sepsis. Contrib Microbiol 17: 48-85, 2011.

10. Simmons GE Jr, Pruitt WM and Pruitt K: Diverse roles of SIRT1 in cancer biology and lipid metabolism. Int J Mol Sci 16: 950-965, 2015.

11. Giblin W, Skinner ME and Lombard DB: Sirtuins: Guardians of mammalian healthspan. Trends Genet 30: 271-286, 2014.

12. Liu TF, Vachharajani V, Millet P, Bharadwaj MS, Molina AJ and McCall CE: Sequential actions of SIRT1-RELB-SIRT3 coordinate nuclear-mitochondrial communication during immunometabolic adaptation to acute inflammation and sepsis. J Biol Chem 290: 396-408, 2015.

13. Xu W, Lu Y, Yao J, Li Z, Chen Z, Wang G, Jing H, Zhang X, Li M, Peng J and Tian X: Novel role of resveratrol: Suppression of high-mobility group protein box 1 nucleocytoplasmic translocation by the upregulation of sirtuin 1 in sepsis-induced liver injury. Shock 42: 440-447, 2014.

14. Jia Y, Zheng Z, Wang Y, Zhou Q, Cai W, Jia W, Yang L, Dong M, Zhu X, Su L and Hu D: SIRT1 is a regulator in high glucose-induced inflammatory response in RAW264.7 cells. PLoS One 10: e0120849, 2015.

15. Gao R, Ma Z, Hu Y, Chen J, Shetty S and Fu J: SirT1 restrains lung inflammasome activation in a murine model of sepsis. Am J Physiol Lung Cell Mol Physiol 308: L847-L853, 2015.

16. Schug TT, Xu Q, Gao H, Peres-da-Silva A, Draper DW, Fessler MB, Purushotham A and Li X: Myeloid deletion of SIRT1 induces inflammatory signaling in response to environmental stress. Mol Cell Biol 30: 4712-4721, 2010.

17. Yang Z, Kahn BB, Shi H and Xue BZ: Macrophage alpha1 AMP-activated protein kinase (alpha1AMPK) antagonizes fatty acid-induced inflammation through SIRT1. J Biol Chem 285: 19051-19059, 2010. 
18. Imai S, Armstrong CM, Kaeberlein $\mathrm{M}$ and Guarente L: Transcriptional silencing and longevity protein Sir2 is an NAD-dependent histone deacetylase. Nature 403: 795-800, 2000

19. Vaquero A, Scher M, Lee D, Erdjument-Bromage H, Tempst P and Reinberg D: Human SirT1 interacts with histone H1 and promotes formation of facultative heterochromatin. Mol Cell 16 93-105, 2004.

20. Liu TF, Yoza BK, El Gazzar M, Vachharajani VT and McCall CE: $\mathrm{NAD}^{+}$-dependent SIRT1 deacetylase participates in epigenetic reprogramming during endotoxin tolerance. J Biol Chem 286 : 9856-9864, 2011.

21. Chen X, Yoza BK, El Gazzar M, Hu JY, Cousart SL and McCall CE: RelB sustains IkappaBalpha expression during endotoxin tolerance. Clin Vaccine Immunol 16: 104-110, 2009.

22. Li J, Qu X, Ricardo SD, Bertram JF and Nikolic-Paterson DJ: Resveratrol inhibits renal fibrosis in the obstructed kidney: Potential role in deacetylation of Smad3. Am J Pathol 177: $1065-1071,2010$

23. El Gazzar M, Yoza BK, Chen X, Hu J, Hawkins GA and McCall CE: G9a and HP1 couple histone and DNA methylation to TNFalpha transcription silencing during endotoxin tolerance. J Biol Chem 283: 32198-32208, 2008.

24. Hou J, Chong ZZ, Shang YC and Maiese K: Early apoptotic vascular signaling is determined by SirT1 through nuclear shuttling, forkhead trafficking, bad, and mitochondrial caspase activation. Curr Neurovasc Res 7: 95-112, 2010.
25. Autiero I, Costantini S and Colonna G: Human Sirt-1: Molecular modeling and structure-function relationships of an unordered protein. PLoS One 4: e7350, 2008.

26. Sharma A, Gautam V, Costantini S, Paladino A and Colonna G: Interactomic and pharmacological insights on human Sirt-1. Front Pharmacol 3: 40, 2012.

27. Kim EJ, Kho JH, Kang MR and Um SJ: Active regulator of SIRT1 cooperates with SIRT1 and facilitates suppression of p53 activity. Mol Cell 28: 277-290, 2007.

28. Li T, Zhang J, Feng J, Li Q, Wu L, Ye Q, Sun J, Lin Y, Zhang M, Huang R, et al: Resveratrol reduces acute lung injury in a LPS-induced sepsis mouse model via activation of SirT1. Mol Med Rep 7: 1889-1895, 2013.

29. Franco JG, de Moura EG, Koury JC, Trotta PA, Cordeiro A, Souza LL, Almeida NA, Lima Nda S, Pazos-Moura CC, Lisboa PC and Passos MC: Resveratrol reduces lipid peroxidation and increases sirtuin 1 expression in adult animals programmed by neonatal protein restriction. J Endocrinol 207: 319-328, 2010.

30. Chen X, El Gazzar M, Yoza BK and McCall CE: The NF-kappaB factor RelB and histone $\mathrm{H} 3$ lysine methyltransferase $\mathrm{G} 9$ a directly interact to generate epigenetic silencing in endotoxin tolerance. J Biol Chem 284: 27857-27865, 2009.

31. El Gazzar M, Yoza BK, Chen X, Garcia BA, Young NL and McCall CE: Chromatin-specific remodeling by HMGB1 and linker histone $\mathrm{H} 1$ silences proinflammatory genes during endotoxin tolerance. Mol Cell Biol 29: 1959-1971, 2009. 